\title{
Genes de radio resistencia en cáncer cérvico-uterino localmente avanzado
}

\section{Artículo de revisión}

\author{
Vallín-Delgado Eder*, Alatriste-Martínez Sara**, Rosas-Cabral Alejandro***
}

\begin{tabular}{|c|c|}
\hline Resumen & Abstract \\
\hline $\begin{array}{l}\text { La radioterapia constituye la piedra angular del tratamiento del cán- } \\
\text { cer cérvico-uterino (CaCu) avanzado. Actualmente, el consenso mun- } \\
\text { dial del tratamiento estándar, de las etapas localmente avanzadas } \\
\text { de esta neoplasia, es la asociación de radioterapia concomitante con } \\
\text { la quimioterapia basada en platino. Se ha demostrado que la expre- } \\
\text { sión de algunos genes dentro de las poblaciones celulares del CaCu } \\
\text { contribuye a la falta de respuesta al tratamiento con quimio-radiote- } \\
\text { rapia, en pacientes con similares factores de riesgo al momento del } \\
\text { diagnóstico. A este grupo de genes se les ha denominado genes de } \\
\text { radio-resistencia o quimio-resistencia. Dentro de estos genes desta- } \\
\text { can los relacionados con la promoción del crecimiento celular indife- } \\
\text { renciado, el escape a los puntos de restricción del ciclo celular y los } \\
\text { relacionados con alteraciones de la apoptosis. Los genes que mayor } \\
\text { interés han despertado hasta el momento como potenciales marca- } \\
\text { dores de radio-resistencia son: BCl-2, Lgals, iASPP, Np73, XCRR5, HIF- } \\
\text { I } \alpha \text {, IGFI, IGF-IR, Bak LDHA, y EIF4A I. Sin embargo, dentro de estos } \\
\text { mecanismos también interfieren otros genes capaces de generar } \\
\text { radio-sensibilidad como los son el RBPI y el ALDHI. Este último, par- } \\
\text { ticularmente, es capaz de generar quimio-resistencia. En el presente } \\
\text { trabajo realizamos una revisión de la evidencia disponible hasta el } \\
\text { momento en la literatura, para conocer los mecanismos a través de } \\
\text { los Cuales actúan estos genes y cómo los mismos pudieran ser mar- } \\
\text { cadores que ayuden a planear una correcta estrategia terapéutica } \\
\text { y, de esta manera, mejorar el pronóstico de las pacientes con CaCu. } \\
\text { LUX MÉDICA AÑ0 15, NÚMER0 43, ENERO-ABRIL 2020. PP 35-47 }\end{array}$ & $\begin{array}{l}\text { Radiotherapy is the cornerstone of advanced cervical cancer } \\
\text { treatment. Currently, the global consensus for the standard treatment } \\
\text { of locally advanced stages is the association of concomitant radiothe- } \\
\text { rapy with platinum-based chemotherapy. It has been shown that the } \\
\text { expression of some genes within the cervical cancer cell populations, } \\
\text { contributes to the lack of response to chemo-radiotherapy treatment } \\
\text { in patients with similar risk factors at the time of diagnosis. This group } \\
\text { of genes has been called radio-resistance or chemo-resistance genes. } \\
\text { These genes include those related to the promotion of undifferentia- } \\
\text { ted cell growth, escape from the cell cycle restriction points, and those } \\
\text { related to apoptosis alterations. The genes that have aroused the } \\
\text { greatest interest so far as potential radio-resistance markers are: BCl- } \\
2 \text {, Lgals, iASPP, Np73, XCRR5, HIF-I } \alpha \text {, IGFI, IGF-IR, Bak LDHA, and } \\
\text { EIF4AI. However, among these mechanisms, there are other genes } \\
\text { capable of generating radio-sensitivity, such as RBPI and ALDHI. } \\
\text { The latter can generate chemo-resistance. In this work, we review the } \\
\text { available evidence in the literature to know the mechanisms through } \\
\text { which these genes act and how they could be markers that help plan } \\
\text { a correct therapeutic strategy, to improve the prognosis of cervical } \\
\text { cancer patients. LUX MÉDICA AÑO 15, NÚMERO 43, ENERO-ABRIL } \\
\mathbf{2 0 2 0 . P P ~} \mathbf{3 5 - 4 7}\end{array}$ \\
\hline $\begin{array}{r}\text { Palabras clave: Cáncer cervico-uterino, radioterapia, } \\
\text { radio-resistencia, XCRR5, HIF-1 } \alpha \text {, IGF1, IGF-1R, Bak, } \\
\text { LDHA, EIF4A1, RBP1, ALDH1 }\end{array}$ & $\begin{array}{l}\text { Keywords: cervical cancer, radiotherapy, radio-resistance, } \\
\text { XCRR5, HIF-1 } \alpha, \text { IGF1, IGF-1R, Bak, LDHA, EIF4A1, RBP1, } \\
\text { ALDH1 }\end{array}$ \\
\hline
\end{tabular}

\footnotetext{
* Médico Pasante de Servicio Social, Unidad Médico Didáctica, Centro de Ciencias de la Salud, Universidad Autónoma de Aguascalientes. México ** Médico Radio-Oncóloga, Corporación Oncológica México Americana SA, Aguascalientes, Ags., México

*** Profesor Investigador, Departamento de Medicina, Centro de Ciencias de la Salud, Universidad Autónoma de Aguascalientes México
}

Fecha de recibido: 2 de octubre 2019

Fecha de aceptado: 6 de diciembre 2019

Correspondencia: Dr Alejandro Rosas Cabral. Departamento de Medicina Edificio 107, planta alta, Centro de Ciencias de la Salud. Universidad Autónoma de Aguascalientes. Avenida Universidad \#940. Teléfono 4499108443 . Código postal 20131. Correo electrónico drrosaascabral@gmail. com 


\section{Introducción}

En el ámbito mundial, el cáncer del cuello uterino $(\mathrm{CaCu})$ es la segunda neoplasia más frecuente y la principal causa de mortalidad en las mujeres de los países en desarrollo. ${ }^{1}$ En México, de acuerdo con el INEGI, el cáncer de órganos genitales femeninos (que incluye al $\mathrm{CaCu}$ y al cáncer de ovario) se ubica desde el 2006 como la segunda neoplasia maligna más frecuente, siendo además la principal causa de muerte por tumores malignos en la mujer; en el 2017 produjo uno de cada 10 fallecimientos por cáncer. ${ }^{2}$ En Aguascalientes, según el Instituto de Servicios de Salud del Estado de Aguascalientes (ISSEA), ${ }^{3}$ en el 2017 la tasa de mortalidad por $\mathrm{CaCu}$ fue de 9.5 por cada cien mil habitantes, de los cuales el $20.3 \%$ se detecta en etapas tempranas, el $18.4 \%$ en una etapa intermedia y el resto en etapas tardías $(61.3 \%)$, siendo la edad promedio al diagnóstico de 49.3 años.

El tratamiento concomitante de quimioterapia (generalmente cisplatino) asociada a la teleterapia y a la braquiterapia intracavitaria constituye el pilar para el $\mathrm{CaCu}$ localmente avanzado. Es decir, las etapas IIB, III, y IVA de la Federación Internacional de Ginecología y Obstetricia (FIGO), así como una alternativa al tratamiento quirúrgico en las etapas IB y IIA. ${ }^{4}$ Sin embargo, se ha observado que el 30 y el $40 \%$ de las pacientes con CaCu localmente avanzado, con factores pronósticos similares al momento del diagnóstico, no responden de la misma manera a este tratamiento estándar ${ }^{5,6}$ y que, además, presentan un riesgo de recurrencia del 50\% que podría ser explicado por la presencia de células radioresistentes en la masa tumoral. ${ }^{7}$ La elevada tasa de síntesis de ADN asociada al extenso daño genético, derivado de sucesivas mutaciones producidas en cada ciclo celular en las células tumorales del $\mathrm{CaCu}$, explicaría la generación de alteraciones en los mecanismos de reparación del ADN que contribuyen a la síntesis descontrolada de este ADN erróneo lo que, a su vez, podría dotar a las células tumorales de resistencia a la quimioterapia y a la radioterapia durante la progresión misma del tumor.

En el 2002, Kitahara y cols. ${ }^{8}$ identificaron, mediante microarreglos, 50 genes cuya expresión en células radioresistentes era significativamente mayor que en células radiosensibles, y los postularon como posibles marcadores predictores de la respuesta al tratamiento y pronóstico de las pacientes con $\mathrm{CaCu}$ localmente avanzado y tratado exclusivamente con radioterapia. Estos genes fueron clasificados de acuerdo a los mecanismos a través de los cuales actúan: 1) genes involucrados en la reparación del $A D N, 2)$ genes codificantes de factores para la duplicación, 3) genes codificantes de factores para la transcripción, 4) genes codificantes de factores para la traducción, 4) genes implicados en la glucólisis, 5) genes del citoesqueleto, 6) genes implicados en la respuesta inmune, 7) genes implicados en la proteólisis, 8) genes relacionados con la apoptosis $y, 9)$ genes diversos. De la misma manera, se han publicado diver- 


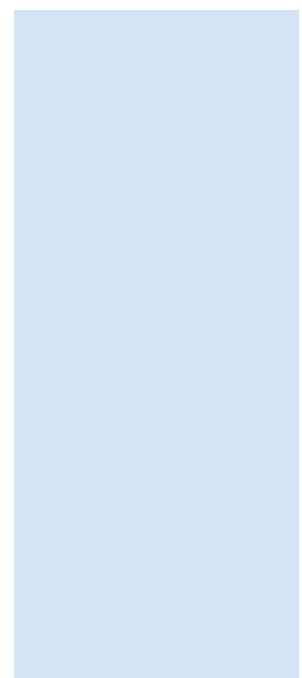

sos trabajos reportando diferentes proteínas detectadas mediante inmunohistoquímica como predictores relevantes de radioresistencia en pacientes con $\mathrm{CaCu}$, al igual que genes sobre-expresados o desregulados en estudios de transfección génica en líneas celulares o animales de laboratorio.

El objetivo de este trabajo fue detallar los mecanismos a través de los cuales la expresión de una selección de proteínas y genes considerados por la literatura más reciente como los más relevantes a este respecto (los genes XCRR5, HIF-1 $\alpha$, IGF1, IGF-1R, Bak, LDHA, EIF4A1 y las proteínas Bcl-2, Lgals, iASPP, y $\triangle$ Np73) contribuyen de manera determinante al desarrollo de radioresistencia en las células tumorales del $\mathrm{CaCu}$. También se abordarán los mecanismos a través de los cuales la expresión del gen RBP1 genera radiosensibilidad en las células tumorales y el doble papel del gen ALDH1 como marcador de radiosensibilidad y quimiorresistencia.

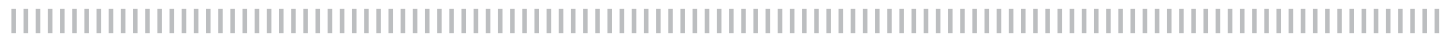

\section{Gen XCRR5}

La elevada tasa de síntesis de ADN asociada a un extenso daño genético derivado de sucesivas mutaciones propias de las células tumorales sugiere la existencia de alteraciones en los mecanismos de reparación del ADN que contribuyen a la síntesis descontrolada de ADN erróneo. Evidencia creciente indica que las células tumorales podrían adquirir resistencia a la quimio y radioterapia durante la progresión misma del tumor modulando la actividad de la maquinaria para la reparación del ADN a través de diversos factores, siendo uno de los más estudiados la proteína Ku80, ya que su presencia se ha demostrado hasta en el $70 \%$ de las células tumorales de pacientes con cáncer cervicouterino ${ }^{9}$ y cuyo nivel de expresión se ha propuesto como marcador predictivo de respuesta a la radioterapia y de supervivencia.

El producto del gen XCRR5 ( $X$-Ray Repair Cross-Complementing 5), la proteína Ku80, constituye, junto con la proteína $\mathrm{Ku} 70$, un heterodímero denominado " $\mathrm{Ku}$ " el cual se une con la región terminal libre del ADN e interactúa directa e indirectamente con varios factores y enzimas relacionadas al mecanismo de reparación tipo NHEJ9 (Non-homolougus end joining), el cual es el principal mecanismo para la reparación de las rupturas en doble cadena del ADN o DSBs generadas por la radioterapia). Por ejemplo, funciona como el componente de unión de la enzima DNAPK (DNA dependent protein kinasa) con el ADN dañado, lo que activa el componente catalítico de la DNA-PK que fungiría, junto con $\mathrm{Ku}$, como el principal estabilizador del complejo de reparación NHEJ. ${ }^{10}$ Además, se ha demostrado que Ku recluta otros factores como fosfatasas, nucleasas, polimerasas, helicasas, fosfodiesterasas y la DNA ligasa IV necesarios para la generación de terminaciones compatibles en las hebras de ADN para su posterior ligamiento ${ }^{11} \mathrm{y}$ que, adicionalmente, evitaría la degradación mediada por nucleasas de las DSBs.

\section{Gen HIF1- $\alpha$}

La hipoxia es una característica importante de los tumores sólidos como el $\mathrm{CaCu}$; se ha observado que hasta el $50-60 \%$ de los tumores localmente avanzados contienen regiones hipóxicas, ${ }^{12}$ en las cuales la $\mathrm{pO}_{2}$ puede alcanzar valores menores a $10 \mathrm{mmHg}$ (valores menores a $12.5 \mathrm{mmHg}$ no se encuentran en tejidos normales). ${ }^{13-14}$ La hipoxia es el resultado tanto de la difusión limitada de oxígeno como de la alta 
tasa metabólica de las células tumorales (hipoxia crónica) o de la oclusión temporal de la vasculatura tumoral malformada (hipoxia aguda). ${ }^{15}$

Se ha demostrado que la expresión del gen HIF- $1 \alpha$ en cáncer cervical se relaciona de manera inversa con la sobrevida a 5 años de las pacientes con $\mathrm{CaCu}$ debido a sus efectos adversos sobre la radioterapia y de manera directa, con el riesgo de muerte relacionada al tumor, de recurrencia local y de generación de metástasis a distancia. La expresión y estabilidad de este factor se favorece en condiciones de hipoxia (en condiciones de normoxia, el HIF1- $\alpha$ es inactivado en 5-8min por hidroxilación de oxígeno- dependiente a través de las enzimas PHD o prolyl-hydroxylase domain enzimes) ${ }^{15-16} y$, además, también es promovida por la misma radioterapia a través de un mecanismo denominado "reoxigenación," en el cual la destrucción de células tumorales lleva a un incremento transitorio de la $\mathrm{pO}_{2}$ que intensifica el estrés oxidativo por el mecanismo de hipoxia-reperfusión, que a su vez, estabiliza directamente al HIF1- $\alpha$ (se ha demostrado que la actividad de este factor empieza a incrementarse aproximadamente 12-24 horas después de la radiación, alcanzando su pico a las 48 horas y prolongándose hasta por una semana). 17,18 Por último, se ha descubierto que las proteínas E6 y E7 del virus del papiloma humano (HPV) son capaces de promover la expresión de HIF-1 $\alpha .^{12}$

Una vez estable, el HIF1- $\alpha$ interactúa con el HIF-1ß formando el complejo HIF-1 que se acopla al factor trascripcional HRE (hipoxia-responsive element) para, posteriormente, traslocarse al núcleo donde controla la expresión de más de 100 genes que en conjunto tienen un impacto negativo en la radiosensibilidad del tumor; ${ }^{19}$ inhiben la señalización para la apoptosis, inducen activación de glucólisis con la producción de elevadas cantidades de piruvato y lactato (a través de los transportadores GLUT 1 y 2, la histidina-quinasa, la aldola- sa, la gliceraldheido-3-fosfato deshidrogenasa y la fosfoglicerato-quinasa-1), activan la producción de lactato (por la lactato deshidrogenasa $A$ ), promueven la angiogénesis (mediante el factor de crecimiento del endotelio vascular o VEGF, el factor transformador del crecimiento- $B$ y el factor de crecimiento derivado de las plaquetas), la invasión y metástasis (a través del factor de crecimiento de los hepatocitos, el CXCR4, las queratinas 14/18/19, metaloproteinasa de la matriz 2 o $M M P 2$, la vimentina, la fibronectina y la catepsina D).

\section{Gen IGF-1}

El factor de crecimiento similar a la insulina 1 (IGF-1) es un pequeño polipéptido que circula en concentraciones relativamente altas en el plasma (150-400 ng/ $\mathrm{ml}$ ), predominantemente unido a proteínas (IGF-binding proteins o IGFBPs), siendo la fracción libre activa menos del $1 \% .{ }^{20}$ Su estructura consiste en dominios homólogos a las cadenas $A$ y $B$ de la insulina que se mantienen unidos a un péptido $C$ análogo al de la insulina y un pequeño dominio adicional $D$ no encontrado en la insulina. ${ }^{21}$

Se ha demostrado que el IGF-1 participa en el proceso de carcinogénesis al incrementar la expresión de la ciclina D1 y la CDK4 que, a su vez, lleva a la fosforilación de RB y a la consecuente activación de la ciclina E1. ${ }^{20-22}$ Además, IGF1 media la activación de CDK4 través de la reducción en la actividad del inhibidor tanscripcional p27/KIP1. En cuanto a su papel en los mecanismos de radiorresistencia, se ha documentado que posee acción sinérgica con el HIF-1 promoviendo la expresión de los factores relacionados con la replicación, angiogénesis, invasión y metástasis tumoral principalmente el VEGF y las metaloproteinasas de la matriz MMP2 y MMP9. ${ }^{20}$ Asimismo, se ha observado que el IGF-1 inhibe a través de diversos factores la vía de señalización de la apoptosis. 
Por último, en otros estudios se ha observado también que el IGF1 participa en el proceso de carcinogénesis al incrementar la expresión de las ciclinas D1 y CDK4, que a su vez llevan a la fosforilación de la proteína RB y la consecuente activación de la ciclina $E$, lo que permite la continuación del ciclo celular a pesar de los errores en el material genético de las células tumorales. ${ }^{21}$

\section{Gen IGF-1R}

El receptor del factor de crecimiento semejante a la insulina 1 (IGF-1R) es un receptor transmembranal constituido por un homodímero con dos subunidades $\alpha$ extracelulares y dos subunidades $\beta$ transmembranales unidas por puentes disulfuro. La subunidad $\alpha$ contiene el dominio de unión al ligando (principalmente IGF-1 y con menor afinidad a IGF-2 e insulina), mientras que la subunidad $B$ posee un dominio transmembranal, un dominio intracelular con actividad de tirosin-kinasa y un dominio C-terminal con funciones de interacción con proteínas. ${ }^{23}$ Una vez activado, dispara cascadas de señalización al núcleo celular modulando algunas funciones celulares a través de la regulación de factores de transcripción; entre las principales vías activadas por este receptor se encuentran la de las MAPK (mitogen-activated protein kinases) y la del PI3K (phosphatidylinositol 3-kinase). ${ }^{6}$

La vía de las MAPK promueve la proliferación y diferenciación celular a través de la activación de la proteína Shc (SRC homology and collagen) que, a su vez, activa múltiples kinasas como Ras, Raf y MEK que participan en diferentes vías estimulando la proliferación celular. Por otro lado, la activación del IGF-1R inhibe la vía apoptótica al activar a la proteína IRS-1 (insuline receptor substrate) que, de igual manera, interactúa con p-85 para la activación de la PI3K disparando la vía de señalización mediada por fosfolípidos activados que a través de una quinasa dependiente de PI3 activa a la Akt (protein kinasa $B$ ) la cual in- teractúa con el complejo $\mathrm{BCL}-2 / \mathrm{BAD}$, inactivando BAD, disociando el complejo y por tanto liberando la proteína antiapoptótica BCL-2.24,25

La activación del IGF-1R también se ha asociado con la pérdida de la adhesión celular (a través de la disrupción de los complejos ß-caderina/E-caderina, favoreciendo la liberación celular), con la motilidad celular (mediante vías que llevan a la reorganización de la actina y la contractilidad actina/miosina), y con la metástasis tumoral (a través de la secreción a la matriz extracelular de anexina II que promueve la activación del plasminógeno y la adhesión celular). ${ }^{25}$

Múltiples estudios han demostrado el valor pronóstico del IGF-1R en la respuesta al tratamiento con radiación; por ejemplo, en embrio-fibroblastos de ratón, aquellas células que no expresan el IGF-1R presentan mayores tasas de apoptosis inducida por radiación, mientras que aquellas que sobre-expresan este receptor tienen inhibido este mecanismo. ${ }^{25}$

\section{Gen Bak}

La familia de las "proteínas relacionadas a BCL-2" constituye el conjunto de genes más relevantes encargados de la regulación biológica de la apoptosis, cuyos productos tienen la capacidad de fungir tanto como antagonistas (Bcl-2, Bcl-KL Bcl-w, Bfl-1/A1, y $\mathrm{Mcl}$-1) como agonistas (Bax, Bak, Bok, Bcl-XS, Bad, Bid, Bik, Bim, Noxa, y Hrk) de esta vía de muerte celular. En el 2001, Chung y cols. ${ }^{26}$ determinaron que sus niveles de expresión se correlacionaban con el grado patológico, la etapa y el estado clínico del $\mathrm{CaCu}$ al momento del diagnóstico, siendo su expresión particularmente común en aquellos tumores pobremente diferenciados; además, encontraron una correlación significativa entre su nivel de expresión y los niveles caspasa 3, como indicador de la activación final de la apoptosis, en las células tumorales posteriores a la radiación. 
Aunado a esto, se ha evidenciado que el incremento en la expresión de Bax (otro factor inhibidor de la apoptosis) después del tratamiento con radioterapia en pacientes con $\mathrm{CaCu}$ se correlaciona con un mejor pronóstico en comparación con aquellas que, después de la radiación, presentaban una pobre expresión del mismo. ${ }^{27,28}$ Esta premisa que, debido la similitud en la estructura y función de Bax y Bak, podría ser aplicada hipotéticamente a este último. Finalmente, otros estudios han observado que, tanto la mutación del gen Bak $^{29}$ como la reducción en su expresión (ya sea por mutación o por su degradación por la proteína E6 del virus del papiloma humano), ${ }^{30,31}$ se relacionan con una menor respuesta tumoral a la radiación.

\section{Gen EIF4A 1}

El factor de iniciación eucariótico 4F (EIF4F) es un complejo heterotrimérico compuesto de la helicasa de ARN dependiente de ATP EIF4A1, la proteína fijadora de mRNA EIF4E y la proteína de andamio EIF4G1.32 En el 2014, Liang y cols. ${ }^{33}$ encontraron que los tres componentes del complejo EIF4F se encontraban sobre-expresados en el $\mathrm{CaCu}$ y que su nivel de expresión se correlacionaba con el estadio de la enfermedad, el tipo histológico, la presencia de metástasis a ganglios linfáticos, invasión parametrial, e invasión estromal profunda. También encontraron que los niveles de expresión de estos componentes variaban tras el tratamiento con braquiterapia. Sin embargo, se observó que sólo los cambios en el nivel de expresión de EIF4A1 eran significativamente relevantes para el pronóstico del $\mathrm{CaCu}$ ya que aquellas pacientes que no presentaron disminución en la expresión de este factor, presentaron una respuesta incompleta a la braquiterapia y una menor sobrevida.

Aunque los mecanismos a través de los cuales actuaría EIF4A1, a este respecto, aún son inciertos, podría ser debido a la activación de los mecanismos para la re- generación, invasión, angiogénesis y metástasis celular posterior al tratamiento con radiación ${ }^{33,34}$ ya que, como se mencionó, EIF4A1 es una helicasa dependiente de ATP y es indispensable para la traducción del mARN, especialmente para aquellos con amplias regiones 5'UTR (untranslated region ó untranslated trailer, regiones no traducidas de los genes) de los cuales dos terceras partes codifican para oncoproteínas o proteínas implicadas en el crecimiento, muerte y proliferación celular. Dentro de éstos se encuentran el mRNA de $\mathrm{N}$ RAS, BCL232 y VEGF. ${ }^{35}$ Por lo tanto, EIF4A1 es considerada una proteína precursora de cáncer y un importante marcador pronóstico y de respuesta al tratamiento. ${ }^{36}$

\section{Gen LDHA, lactato y radio resistencia}

La transformación de las células normales a tumorales conlleva la generación de un switch metabólico caracterizado por el incremento en los requerimientos de glucosa y la consecuente sobreproducción de lactato en condiciones de normoxia en respuesta a las elevadas demandas de ATP y que es consecuencia de defectos en la respiración celular, activación de oncogenes y la sobre-expresión de enzimas glucolíticas y proteínas transportadoras que se conoce como efecto Warburg. ${ }^{37}$ Algunos de los genes relacionados en este cambio de la fosforilación oxidativa por una glucólisis alterada son: myc (que provoca la sobreexpresión de la lactato deshidrogenasa $A$ (LDHA)), NF-kß, Akt/proteín kinasa B, factor de crecimiento epidérmico (EGF), factor de crecimiento semejante a la insulina 1 (IGF1), fosfatidilinositol 3-kinasa (PIK3), mTOR, el oncogen viral homólogo al sarcoma de rata Kristen (K-RAS), la proteín kinasa activada por AMP (AMPK) y el factor inducible por hipoxia $1 \alpha$ (HIF-1 $\alpha) \cdot{ }^{37-39}$

Como se comentó anteriormente en este artículo, el HIF promueve la expresión de múltiples agentes relacionados a esta glucólisis alterada como los transportadores GLUT1 y MCT-4 que garantizan el 
adecuado aporte de glucosa a la célula y la secreción del lactato acumulado al exterior respectivamente $y$, además, también induce la sobreexpresión de la lactato deshidrogenasa A (LDHA), encargada de la conversión de piruvato a lactato que permite la continua generación de NAD+ necesario para diferentes enzimas metabólicas como la GAPDH, con lo que se perpetua la glucólisis aeróbica, la producción de ATP y la generación del lactato. ${ }^{39,40}$

La LDH es un tetrámero compuesto de dos diferentes subunidades, LDHA y LDHB, que pueden ensamblarse en 5 diferentes combinaciones (la LDH1 está compuesta de 4 subunidades LDHB, la LDH2 contiene 3 LDHB y 1 LDHA, la LDH3 contiene 2 LDHA y 2 LDHB, la LDH4 contiene 3 LDHA y 1 LDHB y, la LDH5 contiene 4 subunidades LDHA). ${ }^{39}$ La LDHA tiene mayor afinidad que la LDHB por el piruvato, por lo que la LDH5 es la de mayor importancia para la conversión de piruvato a lactato. El lactato interviene de diferentes maneras en la supervivencia de las células tumorales; activa múltiples mecanismos que contribuyen al escape de las células tumorales de la respuesta inmune, incluyendo la sobreproducción de moléculas inhibitorias y citocinas inmunosupresoras, además de la disminución de la expresión de moléculas co-estimuladoras; inhibe la diferenciación de los monocitos a células dendríticas e inactiva la liberación de sus citocinas y las citocinas de los linfocitos T citotóxicos e induce la muerte por asfixia de los linfocitos $T$ activados al crear un gradiente con alta concentración extracelular de lactato producido por las células tumorales que impide el transporte del lactato producido por las células inmunes al espacio extracelular (que emplean la glucólisis como principal fuente de energía). Induce la producción y activación de factores implicados en la migración y metástasis de las células tumorales como las integrinas B1, el factor transformador del crecimiento ß2 (TGF- B2), el VEGF y activa la producción de ácido hia- lurónico en los denominados fibroblastos asociados al tumor. Por su parte, la LDHA se encuentra elevada en muchos tumores, la mayoría de los cuales poseen altas tasas de glucólisis aeróbica, y altas concentraciones de LDHA se correlacionan con un pobre pronóstico en diferentes tipos de cáncer. ${ }^{39}$

Hirschhaeuser y cols. ${ }^{38}$ demostraron que la acumulación de lactato en CaCu primario se correlacionaba inversamente con la sobrevida de las pacientes y para ello utilizaron la determinación de la enzima LDHA como "sensor" del lactato en las células tumorales postulándola como un posible marcador de mal pronóstico en $\mathrm{CaCu}$; por otro lado Walenta y cols. ${ }^{37-41}$ demostraron en dos diferentes estudios, una correlación positiva entre la cantidad de lactato en las células tumorales y la presencia de metástasis en estadios clínicos tempranos en pacientes con $\mathrm{CaCu}$. Adicionalmente se ha demostrado que las concentraciones de lactato en las células tumorales se correlacionan positivamente con el grado de radiorresistencia tumoral, lo cual podría ser debido a sus propiedades antioxidantes. ${ }^{42-43}$

\section{Papel del gen ALDH1 en quimiorresisten- cia y radiosensibilidad}

La superfamilia enzimática de las aldehído deshidrogenasas humanas (ALDH) está compuesta de 19 isoformas codificadas por genes con distintas localizaciones cromosómicas. Estas enzimas se encuentran en el citosol, núcleo, mitocondria y retículo endoplásmico, donde fungen como enzimas multifuncionales dependientes de $N A D(P)+$ que catalizan la oxidación de varios aldehídos endógenos y exógenos a sus correspondientes ácidos carboxílicos. La ALDH1, además, es capaz de hidrolizar ésteres, unirse a agentes endo y exobióti$\cos$, tiene funciones antioxidantes y cumple roles estructurales; ${ }^{44}$ pero su principal función es la de catalizar la conversión de retinol a ácido retinoico. ${ }^{45} \mathrm{El}$ ácido retinoico 
y sus derivados se relacionan con múltiples mecanismos relacionados con la regulación de la expresión de diversos genes encargados de la proliferación y la diferenciación celular a través de su interacción con sus receptores nucleares. ${ }^{46}$

Se ha demostrado que la expresión ALDH1 se relaciona directamente con el grado de malignidad tumoral al momento del diagnóstico y con la capacidad de auto-renovación y metástasis de las células tumorales del $\mathrm{CaCu} .{ }^{46,47}$ Shu y cols. ${ }^{48}$ y posteriormente Xie y cols. ${ }^{45}$ demostraron, en estudios independientes, que la expresión de ALDH1 previa al tratamiento neoadyuvante con quimioterapia (cisplatino) constituía un factor predictivo independiente en pacientes con $\mathrm{CaCu}$ localmente avanzado y que el incremento en su expresión después del tratamiento con quimioterapia neoadyuvante se asociaba con un mayor riesgo de recurrencia y muerte asociada al tumor.

El mecanismo por el cual la expresión de este gen confiere resistencia a la quimioterapia en las células tumorales aún no se conoce por completo, pero se cree que, al codificar una enzima citosólica, jugaría un rol significativo en la oxidación de aldehídos tóxicos y otros químicos y fármacos potencialmente dañinos como lo son los agentes quimioterápicos.

Aunque no se han dilucidado completamente los mecanismos que relacionan la expresión de la ALDH1 con la radioterapia, se ha observado que niveles de expresión elevados de este gen se relacionan con una mayor tasa de radiosensibilidad; Kitahara y cols. ${ }^{8}$ demostraron que la expresión del gen ALDH1 estaba significativamente incrementada en aquellas pacientes con respuesta completa a la radioterapia en comparación con aquellas que no respondieron al mismo esquema terapéutico, concluyendo, que la expresión elevada de ALDH1, a través del incremento subsecuente en la producción de ácido retinoico, inducía la activación del gen codificante del Ligando inductor de la apoptosis relacionado con el factor de necrosis tumoral (TRAIL), con la subsecuente activación de la vía apoptótica en respuesta a la radiación. De manera similar, también se ha demostrado que la inhibición de la expresión del gen ALDH1 en células madre hematopoyéticas se relaciona con una mayor resistencia de estas a la radiación. ${ }^{49}$

\section{Gen RBP1, ácido retinoico y radiorresistencia}

En los últimos años, diversos estudios se han enfocado en el efecto quimiopreventivo y terapéutico de los retinoides en el $\mathrm{CaCu}$. Algunos estudios han sugerido que los retinoides asociados o no a interferones, son efectivos para la reversión de lesiones premalignas como lo es la neoplasia intraepitelial cervical, lo cual hace pensar que el ácido retinoico podría tener un rol importante en el mantenimiento del epitelio cervical normal. ${ }^{50}$

Los retinoides (derivados naturales 0 sintéticos de la vitamina $A$ ) son potentes reguladores del crecimiento y diferenciación celular y regulan la vía fisiológica para la diferenciación de las células epiteliales. Estos pueden actuar como agentes quimiopreventivos e inhibidores efectivos del proceso de carcinogénesis inducido por químicos o por virus. Los receptores para el ácido retinoico se dividen en tres grupos, $\alpha, \beta, y Y$, los que a su vez tienen diversas isoformas cuya estructura y función depende de su región aminoterminal..$^{50} \mathrm{De}$ todos los receptores para el ácido retinoico, el receptor $ß y$, particularmente la isoforma B2, parece tener un rol dominante como supresor de tumores ya que la disminución en su expresión se ha observado en diversos tumores incluyendo el $\mathrm{CaCu} .^{51}$

Para controlar la cantidad de retinol intracelular, el retinol es convertido a retinal por la enzima alcohol deshidrogenasa $y$, posteriormente, es convertido en tretinoína por la aldehído deshidrogenasa. Adicionalmente, para disminuir el exceso 
de retinol en el citoplasma, se cree que las proteínas fijadores de retinol RBP1 y CRABP1 se asocian con la enzima lecitínretinol aciltransferasa para la esterificación del exceso de retinol en ester de retinil. Tanto RBP1 como CRABP1 pueden actuar como transportadoras de ácido retinoico, acarreándolo del citoplasma al núcleo y facilitando su unión e interacción con sus respectivos receptores..$^{50}$

Se cree que el ácido retinoico actúa como un inhibidor de la carcinogénesis al bloquearla en su fase de promoción, (recordemos que la carcinogénesis consta de 3 fases: iniciación, promoción y progresión) por los siguientes mecanismos: 1) Secuestro celular en la fase $\mathrm{G} 1$ del ciclo celular a través de la expresión del inhibidor de CDK p21 kip1, la degradación proteosómica de la ciclina D1 y la disminución en la producción del mRNA para la CDK2 y ciclina D1. 2) Inducción de la apoptosis a través de la mayor expresión de p21 $1^{\text {Waf1/Cip1 }}$, Bax, CDC2 y Rb2. 3) Inducción de la diferenciación celular a través de mecanismos hasta ahora desconocidos pero que tendrían que ver con la regulación de la expresión y función de distintas kinasas. ${ }^{52}$

Debido a su importancia para la correcta función del ácido retinoico en las células normales y tumorales, se cree que el nivel de expresión de RBP1 podría fungir como marcador de radiosensibilidad en el $\mathrm{CaCu}$; aunque por otro lado se ha demostrado en diferentes estudios que las proteínas fijadoras de retinol (RBP) RBP1 y RBP2 se encuentran sobre-expresadas en pacientes con $\mathrm{CaCu}$ localmente invasor. ${ }^{53}$

\section{BCl-2}

$\mathrm{BCl}-2$ es un oncogen que inhibe la apoptosis inducida por la radiación y por la quimioterapia. ${ }^{54}$ Se ha reportado por diversos autores que la expresión elevada de $\mathrm{Bcl}-2$, produce un incremento en la resistencia a la inducción de apoptosis en cáncer cervical. ${ }^{55}$ Sin embargo, se han reportado resultados contradictorios en el papel de bcl-2 al respecto de la radio resistencia y de la quimio sensibilidad. ${ }^{56}$ Aunado a lo anterior, se ha referido que los tumores que expresan elevados niveles de $\mathrm{Bcl}-2$ tienen resistencia a platino. ${ }^{57}$ Se requieren más estudios para determinar con certeza el papel de bcl- 2 en la radio resistencia del $\mathrm{CaCu}$.

\section{PI3KCA}

Las mutaciones en PI3KCA se han reportado como la alteración genética más frecuente en cáncer cervical. Se considera que el $13 \%$ y el $36 \%$ de los $\mathrm{CaCu}$ presentan mutaciones en PI3KCA. ${ }^{58}$ Este gen codifica para una proteína de 110 kDa, la cual es la subunidad catalítica de la fosfatildilinositol cinasa 3 (PIK3). La vía PIK3/Akt es una de las vías pivote en el desarrollo del cáncer. La unión de un factor de crecimiento con su receptor fosforila y activa al receptor de tirosin cinasa a nivel de la membrana celular. La PI3K fosforila al 4,5 fosfatidil bifosfato a 3,4,5 fosfatidil trifosfato, a su vez fosforila la cinasa dependiente de fosfoinositoles 1(PDK1), que fosforila y activa a Akt. La activación de AKt regula a un grupo de componentes celulares claves en apoptosis, ciclo celular, síntesis de proteínas, reparación de DNA y angiogénesis. La vía PI3K/Akt regula a la enzima proteín cinasa dependiente de DNA (DNA-PK), la cual es responsable de la reparación de las rupturas de doble cadena del DNA causadas por la radiación. Aunado a lo anterior, su inhibición causa una disminución en la regulación de HIF-1 $\alpha$ y del VEGF, lo cual da como resultado disminución de la hipoxia y normalización de la vasculatura. ${ }^{59}$

\section{Galectinas y $\mathrm{CaCu}$}

Las galectinas son una familia de proteínas de unión a ß-galactósidos, las cuales tienen una amplia distribución en tejidos animales, en bacterias y en hongos. Contienen una secuencia altamente conservada de 130 aminoácidos y un dominio de reconocimiento de carbohidratos. Presentan dos características fundamentales: a) Una ele- 
vada similitud en su región conservada, y b) Una elevada afinidad por los ß-galactósidos. La evidencia actual ha relacionado a las galectinas y el desarrollo de cáncer cervical, principalmente en temas relacionados con la formación del tumor, angiogénesis y sensibilidad a la radioterapia, siendo la Gal-1, Gal-3, Gal-7 y Gal-9, que son las principalmente asociadas con alteraciones en estos mecanismos patogénicos. ${ }^{61}$

Kohrenhagen y cols. examinaron la expresión de galectina-1 en 80 biopsias fijadas con parafina: 20 biopsias de tejido cervical sano, 20 de lesión intraepitelial de bajo grado, 20 de lesión intraepitelial de alto grado y 20 de cáncer cervical invasor. Se encontró que la intensidad de la expresión de galectina-1 en las células estromales circundantes a las células trasformadas se incrementaba de acuerdo con el grado patológico, desde tejido cervical benigno, hasta cáncer invasor. Las células epiteliales nunca expresaron galectina-1. Estos hallazgos sugieren que la expresión de galectina-1 en las células estromales del tumor puede ser un marcador del grado histológico del mismo. ${ }^{62}$ También se ha reportado incremento en la expresión de Gal-3, Gal-7 y Gal-9 en tejidos de pacientes con $\mathrm{CaCu}{ }^{63}$

\section{Proteína delta p73 ( $\triangle p 73)$}

La proteína p73 ha sido identificada como un homólogo estructural y funcional de la proteína supresora de tumor p53. Aunque, a pesar de sus semejanzas, estas dos proteínas probablemente ejercen distintas funciones, en particular con relación a la formación del tumor y su progresión. A diferencia del gen p53, que codifica para una sola proteína, el gen p73 codifica para múltiples isoformas de la proteína, debido a splicing alternativo del mRNA. ${ }^{64}$

La forma salvaje de la proteína p73 en su versión completa denominada (TAp73), contiene un dominio amino terminal de transactivación a través del cual puede inducir apoptosis. En contraste, la forma truncada en su dominio amino terminal $(\Delta p 73)$ actúa como un "inhibidor dominante" de TAp73 y p53, con lo cual ejerce un efecto antiapoptótico. Asimismo, al igual que el daño del DNA activa a p53, TAp73 es activada y ésta, a su vez, induce a $\Delta p 73$ y de esta manera puede actuar como un dominante negativo de p53, e inhibir su función. Por lo tanto, el gen p73 puede ser un determinante importante de la respuesta celular a la radiación y la expresión de las dos principales isoformas de su proteína y pueden ser potenciales marcadores para predecir la sensibilidad a la radioterapia en pacientes con cáncer cervical. ${ }^{65}$

\section{iASPP}

La proteína iASPP (inhibidor de apoptosis estimulada por la proteína p53), es codificada por el gen PPP1R13L y se encuentra sobre-expresada en muchos tumores humanos. Es capaz de inhibir la función de p53 a través de la inhibición de la función transactivadora de p53 sobre genes que promueven la apoptosis. El incremento en la expresión de iASPP está asociado con etapas avanzadas, metástasis a ganglios linfáticos, quimioresistencia, radioresistencia y menor sobrevida en pacientes con cáncer cervical y actúa como un promotor clave en la proliferación de las células de $\mathrm{CaCu}$, de la transición epitelial-mesenquimal, invasión y gravedad del mismo. Por lo cual, esta proteína puede ser un marcador de la sensibilidad a la radioterapia en pacientes tratados con quimio-radioterapia concomitante y la inhibición de su función mediante diversas estrategias puede ser relevante para el pronóstico y la respuesta al tratamiento de estas pacientes. 
Existen diferentes vías, a través de las cuales, la expresión de diversos genes altera los mecanismos celulares relacionados con el crecimiento celular, el escape a los puntos de restricción del ciclo celular y la inhibición de la apoptosis promoviendo la generación de colonias celulares radiorresistentes cuya presencia se ha asociado con un pobre pronóstico en las pacientes con $\mathrm{CaCu}$. A la fecha, diferentes estudios continúan en la búsqueda de los mecanismos moleculares a través de los cuales estos genes se relacionan con la radiosensibilidad tumoral con la finalidad tanto de encontrar nuevos objetivos terapéuticos, que pudieran ofrecer oportunidades prometedoras para mejorar la respuesta a la radiación al minimizar los efectos radioprotectores de estos genes, así como para postular a algunos de éstos como potenciales marcadores a determinar al momento del diagnóstico. Todo con el objetivo de identificar oportunamente aquellos tumores radiorresistentes y así implementar esquemas terapéuticos más adecuados para cada paciente y con ello mejorar su pronóstico y posibilidad de curación.

\section{Bibliografía}

1. Benedet JL, Bender $\mathrm{H}$, Jones $\mathrm{H}$ 3erd, Ngan HY, Pecorelli S. FIGO staging classification and clinical practice guidelines in the management of gynecologic cancers. FIGO Committee on gynecologic oncology. Int J Gynaecol Obstet. 2000; 70(2): 209-62.

2. Instituto Nacional de Estadística y Geografía. (2018). Estadísticas a propósito del día mundial contra el cáncer, datos nacionales (4 de febrero) (Comunicado de prensa $\mathrm{N}^{\circ}$ 61/18). Recuperado de http://www.beta. inegi.org. $\mathrm{mx} /$ contenidos/saladeprensa/aproposito/2018/cancer2018_Nal.pdf

3. Centro Nacional de Equidad de Género y Salud Reproductiva. (2017). El cáncer de cuello uterino \#MásCercaDeLoQueParece (Comunicado a propósito de la semana de sensibilización del cáncer de cuello uterino 2017). Recuperado de https://www.gob.mx/ salud\% 7Ccnegsr/acciones-y-programas/semana-desensibilizacion-del-cancer-de-cuello-uterino-2017

4. Vordermark, D. Radiotherapy of Cervical Cancer. Oncology Research and Treatment, 2016; 39(9), 516520. https://doi.org/10.1159/000448902

5. Moreno-Acosta, P., Vallard, A., Carrillo, S., Gamboa, O., Romero-Rojas, A., Molano, M., . . Magné, N. (2017). Biomarkers of resistance to radiation therapy: a prospective study in cervical carcinoma. Radiation Oncology, 12(1). https://doi.org/10.1186/s13014017-0856-2

6. Moreno-Acosta, P., Gamboa, O., Sánchez De Gómez, M., Cendales, R., Díaz, G. D., Romero, A., ... Magné, N. (2012). IGF1R Gene Expression as a Predictive Marker of Response to Ionizing Radiation for Patients with Locally Advanced HPV16-positive Cervical Cancer. Anticancer Research, 32(10), 4319-4326. Recuperado de http://ar.iiarjournals.org/content/32/10/4319. long
7. Pedroza-Torres, A., López-Urrutia, E., García-Castillo, V., Jacobo-Herrera, N., Herrera, L., Peralta-Zaragoza, O., ... Pérez-Plasencia, C. (2014). MicroRNAs in Cervical Cancer: Evidences for a miRNA Profile Deregulated by HPV and Its Impact on Radio-Resistance. Molecules, 19(5), 6263-6281. https://doi.org/10.3390/ molecules19056263

8. Kitahara, O., Katagiri, T., Tsunoda, T., Harima, Y., \& Nakamura, Y. (2002). Classification of Sensitivity or Resistance of Cervical Cancers to lonizing Radiation According to Expression Profiles of 62 Genes Selected by cDNA Microarray Analysis. Neoplasia, 4(4), 295303. https://doi.org/10.1038/sj.neo.7900251

9. Harima, Y., Sawada, S., Miyazaki, Y., Kin, K., Ishihara, H., Imamura, M., . . Ohnishi, T. (2003). Expression of Ku80 in Cervical Cancer Correlates With Response to Radiotherapy and Survival. American Journal of Clinical Oncology, 26(4), 80-85. https://doi. org/10.1097/01.coc.0000077938.48974.59

10. Taccioli, G. E., Gottlieb, T. M., Blunt, T., Priestley, A., Demengeot, J., Mizuta, R., . . Jeggo, P. A. (1994). Ku80: Product of the XRCC5 Gene and Its Role in DNA Repair and V(D)J Recombination. Science, 265(5177), 1442-1445. Recuperado de http://science.sciencemag.org/content/265/5177/1442.long

11. Fell, V. L., \& Schild-Poulter, C. (2015). The Ku heterodimer: Function in DNA repair and beyond. Mutation Research/Reviews in Mutation Research, 763, 15-29. https://doi.org/10.1016/j.mrrev.2014.06.002

12. Liu, J., Zhang, J., Wang, X., Li, Y., Chen, Y., Li, K., . . Guo, G. (2010). HIF-1 and NDRG2 contribute to hypoxia-induced radioresistance of cervical cancer Hela cells. Experimental Cell Research, 316(12), 19851993. https://doi.org/10.1016/j.yexcr.2010.02.028

13. Harada, H., Kizaka-Kondoh, S., Li, G., Itasaka, S., Shibuya, K., Inoue, M., \& Hiraoka, M. (2007). Significance of HIF-1-active cells in angiogenesis and radiore- 
sistance. Oncogene, 26(54), 7508-7516. https://doi. org/10.1038/sj.onc.1210556

14. Harada, H., Inoue, M., Itasaka, S., Hirota, K., Morinibu, A., Shinomiya, K., . . Hiraoka, M. (2012). Cancer cells that survive radiation therapy acquire HIF-1 activity and translocate towards tumour blood vessels. Nature Communications, 3(1). https://doi. org/10.1038/ncomms1786

15. Peitzsch, C., Perrin, R., Hill, R. P., Dubrovska, A., \& Kurth, I. (2014). Hypoxia as a biomarker for radioresistant cancer stem cells. International Journal of Radiation Biology, 90(8), 636-652. https://doi.org/10.3 109/09553002.2014.916841

16. Dewhirst, M. W. (2009). Relationships between Cycling Hypoxia, HIF-1, Angiogenesis and Oxidative Stress. Radiation Research, 172(6), 653-665. https:// doi.org/10.1667/rr1926.1

17. Moeller, B. J., Richardson, R. A., \& Dewhirst, M. W. (2007). Hypoxia and radiotherapy: opportunities for improved outcomes in cancer treatment. Cancer and Metastasis Reviews, 26(2), 241-248. https://doi. org/10.1007/s10555-007-9056-0

18. Meijer, T. W. H., Kaanders, J. H. A. M., Span, P. N., \& Bussink, J. (2012). Targeting Hypoxia, HIF-1, and Tumor Glucose Metabolism to Improve Radiotherapy Efficacy. Clinical Cancer Research, 18(20), 55855594. https://doi.org/10.1158/1078-0432.ccr-120858

19. Harada, H. (2011). How Can We Overcome Tumor Hypoxia in Radiation Therapy? Journal of Radiation Research, 52(5), 545-556. https://doi.org/10.1269/ jrr.11056

20. Clemmons, D. R. (2007). Modifying IGF1 activity: an approach to treat endocrine disorders, atherosclerosis and cancer. Nature Reviews Drug Discovery, 6(10), 821-833. https://doi.org/10.1038/nrd2359

21. LeRoith, D., \& Roberts, C. T. (2003). The insulin-like growth factor system and cancer. Cancer Letters, 195(2), 127-137. https://doi.org/10.1016/s03043835(03)00159-9

22. Samani, A. A., Yakar, S., LeRoith, D., \& Brodt, P. (2007). The Role of the IGF System in Cancer Growth and Metastasis: Overview and Recent Insights. Endocrine Reviews, 28(1), 20-47. https://doi. org/10.1210/er.2006-0001

23. Lloret, M., Lara, P. C., Bordón, E., Pinar, B., Rey, A., Falcón, O., . . . Hernández, M. A. (2007). IGF-1R expression in localized cervical carcinoma patients treated by radiochemotherapy. Gynecologic Oncology, 106(1), 8-11. https://doi.org/10.1016/j.ygyno.2007.04.004

24. Valenciano, A., Henríquez-Hernández, L. A., Moreno, M., Lloret, M., \& Lara, P. C. (2012). Role of IGF-1 Receptor in Radiation Response. Translational Oncology, 5(1), 1-9. https://doi.org/10.1593/tlo.11265

25. Li, R., Pourpak, A., \& Morris, S. W. (2009). Inhibition of the Insulin-like Growth Factor-1 Receptor (IGF1R) Tyrosine Kinase as a Novel Cancer Therapy Approach. Journal of Medicinal Chemistry, 52(16), 4981-5004. https://doi.org/10.1021/jm9002395

26. Chung, T., Cheung, T., Lo, W., Yim, S., Yu, M. Krajewski, S., . . Wong, Y. (2002). Expression of apoptotic regulators and their significance in cervical cancer. Cancer Letters, 180(1), 63-68. https://doi. org/10.1016/s0304-3835(01)00842-4
27. Harima, Y., Harima, K., Shikata, N., Oka, A., Ohnishi, T., \& Tanaka, Y. (1998). Bax and Bcl-2 expressions predict response to radiotherapy in human cervical cancer. Journal of Cancer Research and Clinical Oncology, 124(9), 503-510. Recuperado de https://link. springer.com/article/10.1007\% 2Fs004320050206

28. Qin, C., Chen, X., Bai, Q., Davis, M. R., \& Fang, Y. (2014b). Factors Associated with Radiosensitivity of Cervical Cancer. Anticancer Research, 34(9), 46494656. Recuperado de http://ar.iiarjournals.org/content/34/9/4649.full.pdf + html

29. Wani, K. M. Y., Huilgol, N. G., Hongyo, T., Ryo, H. Shah, K., Chatterjee, N., ... Nomura, T. (2003). Genetic alterations in the coding region of the bak gene in uterine cervical carcinoma. British Journal of Cancer, 88(10), 1584-1586. https://doi.org/10.1038/ sj.bjc. 6600944

30. Cheung, T., Chung, T. K., Lo, K. W., Yu, M., Krajewski, S., Reed, J. C., \& Wong, Y. (2002b). Apotosis-Related Proteins in Cervical Intraepithelial Neoplasia and Squamous Cell Carcinoma of the Cervix. Gynecologic Oncology, 86(1), 14-18. https://doi.org/10.1006/ gyno.2002.6655

31. Thomadaki, H., \& Scorilas, A. (2006). BCL2 Family of Apoptosis-Related Genes: Functions and Clinical Implications in Cancer. Critical Reviews in Clinical Laboratory Sciences, 43(1), 1-67. https://doi. org/10.1080/10408360500295626

32. Raza, F., Waldron, J. A., \& Quesne, J. L. (2015). Translational dysregulation in cancer: elF4A isoforms and sequence determinants of elF4A dependence. Biochemical Society Transactions, 43(6), 1227-1233. https://doi.org/10.1042/bst20150163

33. Liang, S., Zhou, Y., Chen, Y., Ke, G., Wen, H., \& Wu, X. (2014). Decreased Expression of EIF4A1 After Preoperative Brachytherapy Predicts Better TumorSpecific Survival in Cervical Cancer. International Journal of Gynecological Cancer, 24(5), 908-915. https:// doi.org/10.1097/igc.0000000000000152

34. Spilka, R., Ernst, C., Mehta, A. K., \& Haybaeck, J. (2013). Eukaryotic translation initiation factors in cancer development and progression. Cancer Letters, 340(1), 9-21. https://doi.org/10.1016/j.canlet.2013.06.019

35. De la Parra, C., Walters, B. A., Geter, P., \& Schneider R. J. (2018). Translation initiation factors and their relevance in cancer. Current Opinion in Genetics \& Development, 48, 82-88. https://doi.org/10.1016/j. gde.2017.11.001

36. Liang, S., Ju, X., Zhou, Y., Chen, Y., Ke, G., Wen, H., \& Wu, X. (2017). Downregulation of eukaryotic initiation factor 4A1 improves radiosensitivity by delaying DNA double strand break repair in cervical cancer. Oncology Letters. https://doi.org/10.3892/ ol.2017.7040

37. Walenta, S., \& Mueller-Klieser, W. F. (2004). Lactate: mirror and motor of tumor malignancy. Seminars in Radiation Oncology, 14(3), 267-274. https://doi. org/10.1016/j.semradonc.2004.04.004

38. Hirschhaeuser, F., Sattler, U. G. A., \& Mueller-Klieser, W. (2011). Lactate: A Metabolic Key Player in Cancer. Cancer Research, 71(22), 6921-6925. https:// doi.org/10.1158/0008-5472.can-11-1457

39. Doherty, J. R., \& Cleveland, J. L. (2013). Targeting lactate metabolism for cancer therapeutics. Journal of 
Clinical Investigation, 123(9), 3685-3692. https:// doi.org/10.1172/jci69741

40. Yasuda, H. (2008). Solid tumor physiology and hypoxia-induced chemo/radio-resistance: Novel strategy for cancer therapy: Nitric oxide donor as a therapeutic enhancer. Nitric Oxide, 19(2), 205-216. https://doi.org/10.1016/j.niox.2008.04.026

41. Walenta, S., Wetterling, M., Lehrke, M., Schwickert, G., Sundorf, K., Rofstad, E. K., \& Mueller-Klieser, W. (2000). High Lactate Levels Predict Likelihood of Metastases, Tumor Recurrence, and Cancers1 Restricted Patient Survival in Human Cervical. Cancer Research, 60(1), 916-921. Recuperado de http://cancerres.aacrjournals.org/content/60/4/916.full-text.pdf

42. Sattler, U. G., Meyer, S. S., Quennet, V., Hoerner, C., Knoerzer, H., Fabian, C., . . Mueller-Klieser, W. (2010). Glycolytic metabolism and tumour response to fractionated irradiation. Radiotherapy and Oncology, 94(1), 102-109. https://doi.org/10.1016/j.radonc.2009.11.007

43. Shimura, T., Noma, N., Sano, Y., Ochiai, Y., Oikawa, T., Fukumoto, M., \& Kunugita, N. (2014). AKT-mediated enhanced aerobic glycolysis causes acquired radioresistance by human tumor cells. Radiotherapy and Oncology, 112(2), 302-307. https://doi. org/10.1016/j.radonc.2014.07.015

44. Ma, I., \& Allan, A. L. (2010). The Role of Human Aldehyde Dehydrogenase in Normal and Cancer Stem Cells. Stem Cell Reviews and Reports, 7(2), 292-306. https://doi.org/10.1007/s12015-010-9208-4

45. Xie, Q., Liang, J., Rao, Q., Xie, X., Li, R., Liu, Y., . . Lin, Z. (2015). Aldehyde Dehydrogenase 1 Expression Predicts Chemoresistance and Poor Clinical Outcomes in Patients with Locally Advanced Cervical Cancer Treated with Neoadjuvant Chemotherapy Prior to Radical Hysterectomy. Annals of Surgical Oncology, 23(1), 163-170. https://doi.org/10.1245/s10434015-4555-7

46. Yao, T., Wu, Z., Liu, Y., Rao, Q., \& Lin, Z. (2014). Aldehyde dehydrogenase 1 (ALDH1) positivity correlates with poor prognosis in cervical cancer. Journal of International Medical Research, 42(4), 1038-1042. https://doi.org/10.1177/0300060514527060

47. Yao, T., Chen, Q., Zhang, B., Zhou, H., \& Lin, Z. (2011). The expression of ALDH1 in cervical carcinoma. Medical Science Monitor, 17(8), 21-26. https:// doi.org/10.12659/msm.881886

48. Liu, S., \& Zheng, P. (2013). High aldehyde dehydrogenase activity identifies cancer stem cells in human cervical cancer. Oncotarget, 4(12). https://doi. org/10.18632/oncotarget.1578

49. Muramoto, G. G., Russell, J. L., Safi, R., Salter, A. B., Himburg, H. A., Daher, P., . . Chute, J. P. (2010). Inhibition of Aldehyde Dehydrogenase Expands Hematopoietic Stem Cells with Radioprotective Capacity. STEM CELLS. 523-534. https://doi.org/10.1002/ stem.299

50. Abu, J., Batuwangala, M., Herbert, K., \& Symonds, P. (2005). Retinoic acid and retinoid receptors: potential chemopreventive and therapeutic role in cervical cancer. The Lancet Oncology, 6(9), 712-720. https:// doi.org/10.1016/s1470-2045(05)70319-3

51. Ivanova, T., Petrenko, A., Gritsko, T., Vinokourova, S., Eshilev, E., Kobzeva, V., . . Kisseljova, N. (2002). Methylation and silencing of the retinoic acid recep- tor-?2 gene in cervical cancer. BMC Cancer, 2(1). https://doi.org/10.1186/1471-2407-2-4

52. Niles, R. M. (2000). Recent advances in the use of vitamin $A$ (retinoids) in the prevention and treatment of cancer. Nutrition, 16(11-12), 1084-1089. https:// doi.org/10.1016/s0899-9007(00)00436-6

53. SONG, J., LEE, J., LEE, N., JUNG, H., KIM, S., \& LEE, K. (2008). Microarray analysis of normal cervix, carcinomain situ, and invasive cervical cancer: identification of candidate genes in pathogenesis of invasion in cervical cancer. International Journal of Gynecological Cancer, 18(5), 1051-1059. https://doi.org/10.1111/ j.1525-1438.2007.01164.x

54. Reed JC. BCl-2 and the regulation of programmed cell death. J Cell Biol. 1994;124(1-2):1-6.

55. Wootipom V, Lekhyananda N, Phungrassami T, Bonyaphipat $P$, Thongsuksai $P$. Prognostic significance of $\mathrm{BAX}, \mathrm{bcl}-2$, and $\mathrm{p} 53$ expression in cervical squamous cell carcinoma treated by radiotherapy. Gynecol Oncol. 2004;94(3):636-42.

56. Padovan $P$, Salmova $R$, Marchetti $M$, Padovan $R$. Prognostic value of bcl-2, p53, and Ki67 in invasive squamous carcinoma of the uterine cérvix. Eur J Gynaecol Oncol. 2000;21(3):267-272.

57. Kato R, Haegawa K, Torii Y, Udagawa Y, Fukasawa I. Factors affecting pltinum sensivity in cervical cancer. Oncol Lett. 2015:10(6); 3591-3598.

58. Wang J, Chai YL, Wang T, Liu JH, Dai PG, Liu Z. Genetic alterations of PI3KCA and tumor response in patients with locally advanced cervical squamous cell carcinoma treated with cisplatin based concurrent chemoradiotherapy. Exp Mol Pathol. 2015;98(3): 407-410.

59. Arjumad W, Merry CD, Wang C, Saba E, McIntyre JB, Fang S, Kornaga E, Ghatage P, Doll CM, Lees-Miller SP. Oncotarget 2016; 7:82424-82439.

60. Liu FT, Rabinovich GA, Galectins as modulators of tumour progression. Nature Reviews Cancer. 2005; 5 (1): $29-41$.

61. Park JE, Chang WY, Cho M. Induction of matrix metalloproteinase- 9 by galectin-7 through p38 MAPK signaling in HeLa human cervical epithelial adenocarcinoma cells. Oncology Reports. 2009;22(6):13731379.

62. Kohrenhagen N, Volker HU, Kapp M, Dietl J, Kammerer $U$. Increased expression of galectin-1 during the progression of cervical neoplasia. International Journal of Gynecological Cancer. 2006;16(6):2018-2022.

63. Wang $L$, Zhao $Y$, Wang $Y$, Wu $X$. The role of galectins in cervical cancer biology and progression. Biomed Res Int. 2018: 2175927.doi 10.1122/2018/2175927.

64. Moll UM, Erster S, Zaika A. p53, p63 and p73: solos, alliances and feuds among family members. Biochim Biophys Acta. 2001; 1552:47-59.

65. Liu SS, Chang KYK, Cheung ANY, Liao XY, Leung TW, Ngan HYS. Expression of ?Np73 and Tap73? independently associated with radiosensivities and prognoses in cervical squamous cell carcinoma. Clin Cancer Res. 2006; 12(13):3922-7. 